\title{
Photographie et image en prose dans Dora Bruder de Patrick Modiano
}

\author{
Annelies Schulte Nordholt
}

Published online: 4 September 2011

(C) The Author(s) 2011. This article is published with open access at Springerlink.com

\begin{abstract}
In Patrick Modiano's inquiry on Dora Bruder, photographical imagesmostly portraits of Dora and her parents-play an important role. But in the original edition, these photographs are not reproduced, they are only described. We thus have to do with prose images, with an ekphrasis from existing photographs. In later editions-especially the American and Japanese translations-we find reproductions of these photographs. Their addition to the text modifies the status of it, making it into a biography or document. But our analysis concentrates on the prose images themselves: how do they work out within the narrative? How do they affect the experience of the reader, who «sees » them without really seeing them? What is their relation to postmemory? How do they include the viewpoint of the narrator, who is describing the photographs? We will see that the prose image is a deliberate choice of Modiano's, and that it perfectly fits the narrative formula of Dora Bruder, which is halfway between a memorial and an autobiography. Moreover, it is in harmony with Modiano's mode of narration.
\end{abstract}

Keywords Patrick Modiano - Dora Bruder · Photography · Shoah · Postmemory

Comme la plupart des romans précédents de Modiano, Dora Bruder parut d'abord dans la collection blanche de Gallimard (1997). Collection qui propose le texte dans le dépouillement le plus extrême, sans illustrations de couverture ou à l'intérieur. Le lecteur distrait pouvait donc aisément prendre ce livre pour le nouveau roman de Modiano. D'autant plus que le texte semblait construit sur la même formule que les précédents, celle d'une enquête : un narrateur anonyme y poursuit une enquête

\footnotetext{
A. Schulte Nordholt $(\bowtie)$

Département de Langue et Littérature Françaises, Université de Leiden,

PB 9515, 2300 RA, Leiden, The Netherlands

e-mail: a.schultenordholt@hum.leidenuniv.nl
} 
problématique sur une jeune fille disparue pendant l'Occupation, Dora Bruder, dont il ignore tout. Pourtant, pour éviter tout malentendu, l'éditeur avait jugé bon orner la couverture d'un bandeau rouge portant le texte : «Née le 25/02/1926 à Paris, $12^{\mathrm{e}}$ ». Certificat d'authenticité : cette fois-ci, il s'agissait d'une enquête réelle sur un personnage ayant vraiment existé, comme d'ailleurs les interviews avec l'auteur l'ont vite confirmé. A l'avis unanime, Modiano a fait un travail exemplaire de biographe et de «gardien de la mémoire». En réunissant un maximum de documents (extraits de naissance, mains courantes du commissariat de police, circulaires, rapports, fiches du camp de Drancy...), il a érigé un mémorial pour Dora Bruder mais aussi pour des dizaines d'autres Juifs parisiens, qui figurent dans le livre avec leur nom et un abrégé de leur histoire.

Par une technique de collage, ces documents sont insérés dans le récit, se substituant par moments à lui. Au cours de son enquête, Modiano a également mis la main sur des photographies de Dora, or celles-ci ne sont pas insérées dans le récit, elles ne sont pas reproduites mais uniquement décrites en détail. Ce sont des images écrites, des «images en prose », pour reprendre le terme de Marianne Hirsch (1997, p. 3). Par leur caractère ekphrastique, elles diffèrent donc des autres documents que comporte le récit. Dans ce qui suit, nous interrogerons de près ces images écrites : comment fonctionnent-elles à l'intérieur du récit? En quoi l'approche ekphrastique transforme-t-elle fondamentalement ces photos? Que leur ajoute-t-elle? Et enfin, quels motifs Modiano a-t-il pu avoir pour s'en tenir à l'image écrite, au lieu de la reproduction pure et simple? Craignait-il d'enfreindre ce qu'il appelle «le secret» de Dora? La reproduction convenait-elle mal au genre même de son récit? Ou peutêtre la photo écrite était-elle la meilleure manière de présenter et de conserver ces images?

\section{Petite histoire du paratexte}

Avant de passer à l'analyse des photographies écrites dans le récit, il faut s'arrêter un moment au paratexte de Dora Bruder. En effet, alors que la première édition du récit, comme on l'a vu, était privée d'images d'aucune sorte, on voit des photographies apparaître dans les éditions successives-et notamment dans les éditions étrangères. Ces photographies ne sont d'ailleurs pas seulement des portraits de Dora. Ainsi, la deuxième édition, en Folio (Modiano 1999a), porte une photographie de René-Jacques en première de couverture, au lieu d'un dessin de Pierre Le Tan, comme les autres romans de Modiano en Folio (Fig. 1). Cette photographie, c'est l'auteur qui l'a proposée à l'éditeur. ${ }^{1}$ Le choix d'une photographie au lieu d'un dessin est un geste comparable à celui du bandeau rouge de la première édition. Il s'agit là encore d'authentifier le récit, de le rattacher à une réalité historique et même à un lieu précis. Car, on le sait depuis Barthes, toute photographie est un index, une trace tangible, une preuve que son référent a bel et bien existé, dans un espace-temps précis (Barthes 1980). Or on dirait que la photographie de couverture étend ce statut d'index au texte qui suit. En effet, elle

\footnotetext{
1 Précision de l'auteur dans une lettre du ler juillet 2011.
} 
Fig. 1 Couverture de l'édition

Gallimard Folio. Photo

René-Jacques, «Porte de

Clignancourt», hiver 1948.

C Ministère de la Culture,

France

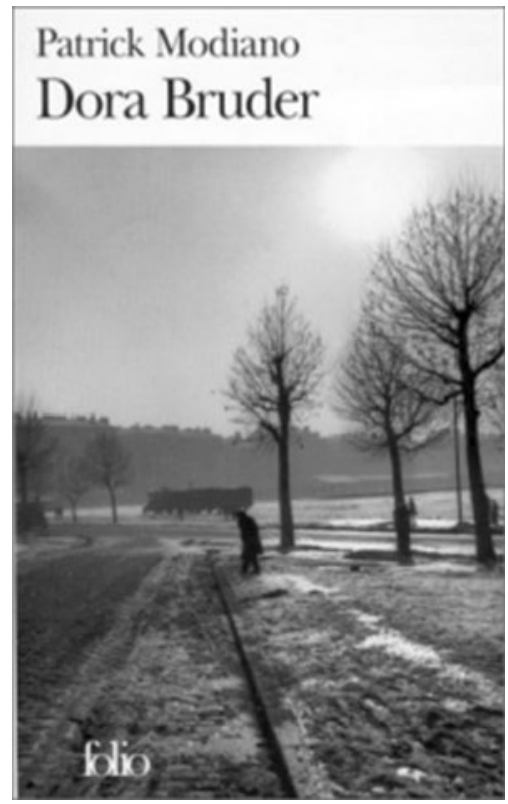

situe implicitement l'histoire de Dora dans l'espace-le paysage urbain de Paris, et même plus précisément, la banlieue-et dans le temps, car la photographie de RenéJacques, en noir et blanc, se réfère sinon au Paris de l'Occupation, du moins à un Paris antérieur à l'explosion urbaine des années 70.

On y voit un paysage sombre, hivernal : arbres dépouillés, flaques. Les quelques passants sont de simples silhouettes. La chaussée inégale débouche sur une route. $\mathrm{Au}$ delà, on voit la silhouette noire d'un véhicule qui pourrait être un wagon de train, ou un omnibus, à l'arrêt. Au fond, en contre-lumière, la silhouette des immeubles. Il est frappant de voir comment, mise en première page de couverture, cette photographie se trouve immédiatement intégrée au roman, dans l'esprit du lecteur, par un effet d'association. Cet effet peut également être trompeur, comme le montre la réaction de Denise Cima par exemple, qui considère la photo comme «emblématique du destin de Dora ». Le train «arrêté dans la neige » et la chaussée qui semble se diriger vers le train, c'est une image qu'elle associe subjectivement à la photographie célèbre de «l'entrée du camp d'Auschwitz, dans laquelle au même emplacement, on voit les rails menant à l'entrée du camp.». La photo évoquerait donc «par association d'idées, la 'voie toute tracée' vers le train, les camps. » (Cima 2003, p. 26). Le spectateur projette ici sur l'image un savoir qui lui est postérieur et en l'occurrence, parfaitement étranger. Du coup, l'histoire de Dora prend une dimension téléologique, comme si c'était son 'destin' d'être persécutée et déportée. La vision de Cima est d'ailleurs sélective, car elle choisit d'ignorer la silhouette des immeubles, qui situe immanquablement cette photo à Paris, aux limites de la ville, dans cette zone floue, ce no man's land entre ville et campagne, qui joue un si grand rôle dans Dora Bruder, comme dans d'autres romans de Modiano. 
Fig. 2 Couverture de l'édition allemande, Carl Hanser, 1998

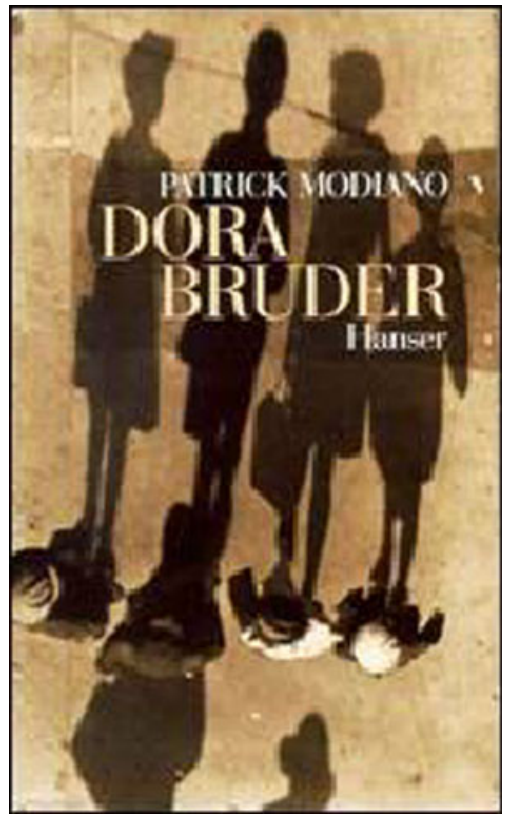

Dès les premières pages, Dora est en effet associée au quartier Clignancourt, où elle a passé son enfance. Et c'est par cette zone de Paris, qu'il a fréquentée lui aussi dans son enfance, que Modiano va tout d'abord se rapprocher d'elle, jusqu'à sentir sa présence diffuse. C'est la première d'une longue série de communions avec le passé qui passent par un lieu, un espace. Ce lieu, c'est ici une zone-limite, une zonefrontière de Paris—la porte de Clignancourt—ou qui du moins l'était dans l'enfance de Dora, et encore vingt ans après, lorsque Modiano enfant la fréquenta, au début des années 60:

A la porte de Clignancourt, le bâtiment et la barrière de l'octroi. A gauche, entre les blocs d'immeubles du boulevard Ney et le marché aux Puces, s'étendait tout un quartier de baraques, de hangars, d'acacias et de maisons basses que l'on a détruit. Vers quatorze ans, ce terrain m'avait frappé. J'ai cru le reconnaitre sur deux ou trois photos, prises l'hiver : une sorte d'esplanade où l'on voit passer un autobus. Un camion est à l'arrêt, on dirait pour toujours. Un champ de neige au bord duquel attendent une roulotte et un cheval noir. Et, tout au fond, la masse brumeuse des immeubles. (Dora Bruder, pp. 34-35). ${ }^{2}$

Le souvenir passe ici par des photographies, encore une fois non reproduites, mais seulement évoquées. Ces photos ne sont certes pas identiques à la photo de couverture de René-Jacques, mais elles lui ressemblent à quelques détails près : ici, c'est non pas un camion mais un wagon de train qui est à l'arrêt (appartenant à la Petite Ceinture, qui passait par là?) La roulotte et le cheval noir sont certes absents, mais on voit la même silhouette des immeubles, en contre-jour. La photo de

\footnotetext{
$\overline{2}$ Toutes les citations sont tirées de l'édition Folio, 1999.
} 
Fig. 3 Couverture de l'édition Gallimard en cd audio (2006): Dora avec son père et sa mère (Dora Bruder pp. 32-33, no. 6). Photo reproduite avec

l'autorisation de P. Modiano

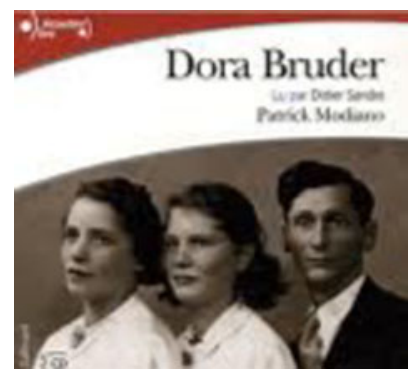

couverture choisie peut donc être considérée comme une référence indirecte à ce passage, peut-être même a-t-elle été choisie parmi une série de photos de la porte de Clignancourt, dont il est question dans ce passage. ${ }^{3}$ Elle vient à point nommé, au seuil du livre, annoncer l'atmosphère hivernale de solitude et de désolation qui marque la double histoire de Dora et de l'auteur.

La même année 1999 voit la parution de la traduction américaine, par Joanna Kilmartin. Ici, le paratexte, et par là même la maquette, prend une place prépondérante. Cela tient certes en premier lieu à la photographie de Dora avec ses parents, reproduite sur la page de garde et dont le détail-le portrait de Dora-se retrouve en filigrane sur la première et sur la quatrième de couverture. Il s'agit du portrait des Bruder en file indienne, qui figure parmi les photographies décrites par Modiano (Fig. 3). Dans cette édition américaine, face aux pages où elles sont décrites, sont reproduites deux autres photos : celle de Dora avec sa mère, posant sur un cube blanc (Fig. 4) et enfin, celle de Dora avec sa mère et sa grand-mère (Fig. 5). Nous reviendrons en détail sur ces photographies, et sur les «images en prose » qui leur correspondent (cf. $\S 3$ et 4). Ce qu'il convient de voir ici, c'est comment elles modifient le statut du livre, le transformant en une biographie, un document. D'autres éléments du paratexte y concourent, dans cette édition, comme les plans $\mathrm{du} 12^{\mathrm{e}}$ et $\mathrm{du} 18^{\mathrm{e}}$ arrondissements, dont le premier est reproduit en filigrane sur la première et quatrième de couverture : non seulement ce livre a pour sujet une personne ayant réellement existé, mais cette vie est liée à un espace urbain fort précis. Par le subtil travail de filigrane, cette maquette suggère l'imbrication étroite de Dora et de son 'habitat' parisien. Enfin, on peut mentionner les mots-clefs figurant sur la page de garde : «1. Modiano, Patrick (1945-). 2. Bruder, Dora, 1926-1942? 3. Holocaust, Jewish (1939-1945). » Le livre est ici classifié comme biographie à sujet historique, et non comme le récit de facture littéraire qu'il est en France.

On voit donc que, très vite après la parution du récit, des photographies-celles de Dora mais aussi des photos du Paris de l'époque-viennent compléter le «dossier Dora Bruder». Ce besoin d'images, c'est le récit lui-même qui le suscite : la ténacité presqu'obsessive de l'enquête de Modiano, sa patiente chasse à la «pièce à conviction », le rôle privilégié de la topographie et des lieux, tout cela incite le lecteur à l'imiter. Et les éditeurs ne font que donner suite à ce désir de la part du

\footnotetext{
${ }_{3}^{3}$ Dans une lettre, l'auteur confirme ce point, en précisant qu'il s'agit d'une photo prise l'hiver 1948, faisant partie d'une série de photos s'intitulant «Porte de Clignancourt» ou «Zone Clignancourt».
} 
Fig. 4 Dora et sa mère (Dora

Bruder p. 32, no. 4). Photo reproduite avec l'autorisation de P. Modiano

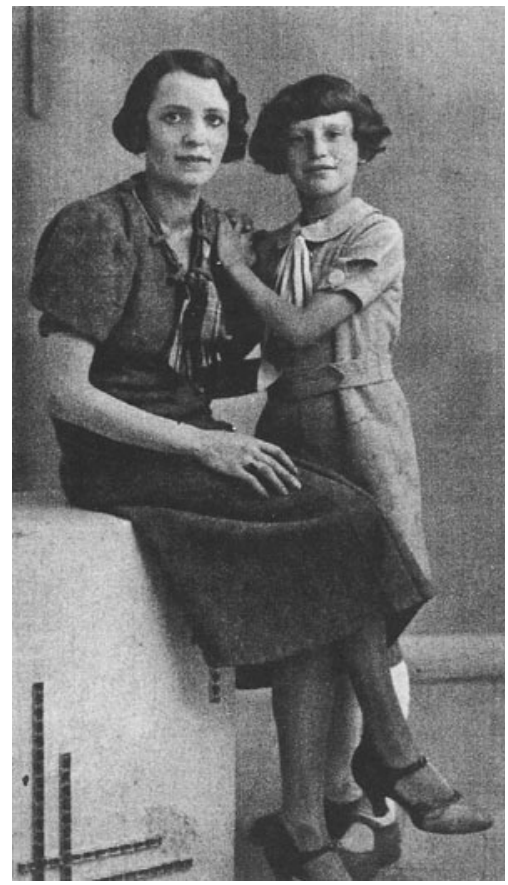

lecteur. Du moins certains, comme l'éditeur japonais, qui publie les mêmes photographies que l'américaine, et les complète par plusieurs autres documents, notamment la reproduction de l'annonce de France Soir. ${ }^{4}$ En 2006, Gallimard publie une édition audio où figure la photographie de Dora avec son père et sa mère (Fig. 3). D'autres restent plus proches de l'édition Folio, et optent, en première de couverture, pour une image ayant un rapport seulement indirect avec le sujet : silhouettes humaines en contre-jour (édition allemande, Fig. 2), passage obscur (édition portugaise)...

\section{Photographie, postmémoire, autobiographie}

Lorsque les éditions américaine et japonaise ont paru, les photographies de Dora étaient déjà connues du public, elles circulaient depuis la parution de la première édition du récit. En effet, dans plusieurs interviews avec l'auteur, au printemps 1997, elles avaient déjà été publiées (Frain 1997; Gaudemar 1997). J'ai analysé ailleurs la genèse complexe du récit, et le rôle capital, mais dérobé, de Serge Klarsfeld dans l'enquête de Modiano, en particulier en ce qui concerne ces photographies (Schulte Nordholt 2008, pp. 96-98). Lorsqu'en 2001, Klarsfeld publie la seconde édition du Mémorial des enfants, Dora Bruder y figure pour la

\footnotetext{
${ }^{4}$ Il s'agit de l'annonce placée par les parents de Dora, en 1941, pour signaler sa fugue; annonce qui a déclenché l'enquête de Modiano (Dora Bruder p. 7). L'annonce est également reproduite en couverture de l'édition de Dora Bruder dans la collection La bibliothèque Gallimard (2004).
} 
première fois (Fig. 3). En quelques lignes, Klarsfeld y rappelle l'origine de cette photo : «Nous avons retrouvé endommagée la photo de Dora Bruder et de ses parents sur une tombe du cimetière de Bagneux; puis grâce à l'une de ses cousines, la photo originale et Patrick Modiano a pu découvrir enfin le visage de Dora.» (Klarsfeld 2001, vol. 4, p. 534). Il ajoute en facsimile l'article que Modiano avait publié dans Libération lors de la parution de la première édition du Mémorial des enfants (Modiano 1994), ainsi que quelques extraits de lettres qu'il lui a adressées au cours des années. Ce dossier confirme ce que Modiano avait souvent déjà affirmé dans ses interviews : que, dès ses lointains débuts, son enquête sur Dora a été soutenue et alimentée par les recherches de Klarsfeld, qui lui a notamment procuré les photographies en question. Inversement, Modiano a partagé avec Klarsfeld les résultats de son enquête, notamment lorsqu'il a découvert la date de naissance de Dora. $^{5}$

Reste qu'à l'intérieur de son récit, Modiano ne cite pas ses sources, ou ne les cite que très vaguement. S'il cite in extenso la mention des Bruder dans le Mémorial de la déportation des Juifs de France de Klarsfeld (1978), tout en en respectant la typographie particulière (Dora Bruder, p. 54), cependant le nom de Klarsfeld n'est pas mentionné. Il en va de même pour d'autres personnes l'ayant aidé à avancer dans son enquête. C'est que, malgré ce que laisse supposer le paratexte de plusieurs éditions étrangères, Dora Bruder n'a jamais voulu être une simple biographie ou un compte rendu d'enquête historique, pourvu de notes en bas de page mentionnant la source précise des documents proposés. C'est un récit, comparable quant à sa formule narrative aux autres récits de Modiano : au centre, un protagoniste-narrateur qui, en véritable détective, mène l'enquête, et la raconte en détail. Cependant, cette fois-ci, ce narrateur coïncide entièrement avec l'auteur lui-même. Comme d'autres l'ont observé (Cima 2003, p. 43), cela implique le choix de la focalisation interne, qui fait que tout est vu et interprété uniquement par ce narrateur. Modiano étant ici à la fois narrateur et écrivain, romancier, il se sert de ses pouvoirs de déduction mais aussi de sa faculté de recréer Dora par l'imagination et le rêve. Cette imagination est déclenchée lorsqu'il s'identifie à elle, associant des pans entiers de la vie de Dora à la sienne propre et à celle de son père.

En effet, comme on sait, le récit contient plusieurs projets commémoratifs : un projet biographique, certes, mais aussi un projet autobiographique, qui mène Modiano sur les traces de son histoire personnelle, notamment celle de son père. En effet, dans sa quête de Dora, le narrateur est plus que jamais à la recherche du passé d'avant sa propre naissance. Comme le narrateur de Livret de famille, il pourrait dire que «ma mémoire précédait ma naissance» (Modiano 2001, p. 116). Sa mémoire est une «postmémoire», selon le terme de Marianne Hirsch : une mémoire par procuration, empruntée aux récits, à la vie des autres, vécue de manière différée, retardée (Hirsch 1996, p. 662). Dora Bruder-comme son nom de Bruder, signifiant «frère » en allemand, l'indique, aux yeux de certains-est dans un certain sens une sœur de Modiano. Elle est son aînée de vingt ans, par le biais de qui, par procuration, il peut vivre l'Occupation, et devenir le contemporain de son propre père, percer le mystère de la survivance de celui-ci pendant les «années sombres ».

\footnotetext{
5 Sur le détail de ces échanges, cf. Morris (2006).
} 
Ce projet autobiographique ne concerne pas seulement le père, mais aussi sa propre enfance et jeunesse. Par un jeu d'empathie extrême, le fil de l'histoire de Dora est constamment interrompu, et complété, par celui de l'autobiographie. Aux lacunes de la biographie de Dora, Modiano supplée par des fragments de sa propre enfance et jeunesse. Cet enchevêtrement constant entre la vie de Dora et la sienne, jusqu'au brouillage, à la «surimpression », indique la grande place de l'autobiographie dans le récit (Schulte Nordholt 2008, p. 104 ss.). Cette place aurait été amoindrie si Modiano avait reproduit les photos de Dora; le récit aurait été réduit à une biographie documentaire.

Enfin, outre le biographique et l'autobiographique, il y a une troisième dimension, celle de la commémoration collective. Par le biais de Dora Bruder, Modiano commémore tous les Juifs parisiens persécutés et déportés. Dans ce sens, comme l'a remarqué Denise Cima, le livre est un 'tombeau' (Cima 2003, p. 27) : en l'honneur de Dora Bruder et de son père, mais aussi de dizaines de Juifs parisiensdes jeunes surtout, comme Dora-qui y figurent avec leur nom et un abrégé de leur histoire. Là aussi, on peut imaginer qu'en reproduisant les photographies de Dora dont il disposait, Modiano eût paru privilégier celles-ci au-dessus des autres. Trois entreprises s'entrelacent donc dans le récit, et se complètent en formant un tissu compliqué. Dans l'histoire de Dora, Modiano perçoit une multitude d'échos : tantôt de sa propre histoire (mêlée à celle de son père), tantôt de l'histoire des compagnons d'infortune de Dora.

Revenons maintenant à notre question initiale : est-ce que le choix pour l'image écrite, ekphrastique, au lieu de la photo reproduite, ne saurait pas lui aussi être mis en rapport avec cette formule narrative particulière, étrangère au document historique, entre biographie et autobiographie?

\section{La photographie en prose et le retour du spectre}

Il y a dans Dora Bruder deux passages composés de photographies en prose, très différents. Le premier décrit en deux pages huit portraits-photos de Dora et de ses parents, datant tous de l'avant-guerre (Dora Bruder, pp. 31-33). Le deuxième passage se trouve une soixantaine de pages plus loin, il se compose d'une seule photographie en prose, celle de Dora avec sa mère et sa grand-mère (ibid. pp. 90-91). Or ce dernier passage est en contraste flagrant avec le premier, l'atmosphère y est toute différente. Autant les photos d'avant-guerre sont empreintes d'innocence et de vie, autant l'autre semble refléter l'atmosphère angoissante de l'Occupation. D'où vient cette différence d'atmosphère? Se trouvet-elle dans les photos elles-mêmes ou vient-elle plutôt du regard de celui qui les décrit? C'est un regard qui analyse, commente, remet en contexte, mais aussi un regard qui rêve sur ces photographies, faisant mille hypothèses à leur propos. Or c'est à travers lui seulement que le lecteur 'voit' les photos, sans les voir réellement...

Un premier caractère de la photographie en prose, c'est qu'elle fait intégralement partie du texte, elle s'y trouve insérée beaucoup plus étroitement que si elle était reproduite. Entre le récit et la photographie en prose, il y a unité stylistique mais 
aussi unité quant au contexte. Ainsi le premier ensemble de photographies survient à un moment précis du récit : Modiano vient de découvrir la date de naissance de Dora, et l'acte de mariage des parents, il s'ingénie maintenant à recueillir quelques bribes des années d'enfance de Dora. Mais il ne réussit à identifier ni l'école primaire qui fut la sienne, ni la profession d'Ernest et de Cécile Bruder, et finit par avouer son ignorance : «Je ne sais rien d'eux, au cours de ces années.» (Dora Bruder, p. 31). Restent des hypothèses sous forme de questions. C'est alors que survient la description des huit photos d'avant-guerre. ${ }^{6}$ Elles viennent donc en quelque sorte se substituer à des données concrètes absentes. Une telle présentation pose une question implicite : est-ce que ces photographies combleront la lacune qu'est l'enfance de Dora?

Voici le début du passage : «Quelques photos de l'époque. La plus ancienne, le jour de leur mariage. Ils sont assis, accoudés à une sorte de guéridon. » (p. 31, no. 1). Ce qui frappe tout d'abord, c'est le brusque passage au présent, car jusqu'ici, toutes les données sur Dora Bruder ont été communiquées à l'imparfait ou au passé simple. Certes, le présent est le mode naturel de description d'une image, mais ici il convient particulièrement bien au mode de vision propre à la photographie. La photographie en effet présentifie, elle restitue un moment du passé (même si, on le sait, elle le restitue comme absent). C'est pour cela qu'elle est un outil privilégié de la postmémoire, qui vise à recréer, à faire revivre le passé, et non pas à l'évoquer comme une ère révolue.

Ce qui frappe aussi dans cette description, c'est son caractère décousu, qui est causé par deux éléments stylistiques. Premièrement, les photos sont introduites par des phrases elliptiques, souvent sans verbe : «Une photo avec leur fille Dora. Ils sont assis, Dora est debout entre eux : elle n'a pas plus de deux ans. Une photo de Dora, prise certainement à l'occasion d'une distribution des prix. [...] Une autre photo, prise dans le même lieu [...] » etc. (je souligne). Comme on voit, d'une photo à l'autre, il n'y a aucun mot de liaison; il n'y a pas non plus de marque typographique de transition : le texte se présente presque en un seul bloc, sauf pour le dernier paragraphe, où on passe à la ligne. Ainsi, les photos-dont certaines se ressemblent un peu-s'accumulent, provoquant un sentiment de vertige chez le lecteur. Enumérées de telle façon, elles ne font pas série, formant tout au plus un ensemble d'éléments en vrac, d'instantanés sans continuité. En effet, leur ordre n'est chronologique qu'en apparence : certes, la première est la photo de mariage des Bruder, suit une photo de la petite enfance, puis trois photos de l'enfance, une de l'adolescence, mais les deux dernières reviennent en arrière, vers une Dora enfant, donnant une structure cyclique à la description. Elles ne prétendent donc raconter aucune 'histoire' de Dora et de sa famille.

Quel 'ordre' pourrait-on donc y discerner? Cet ordre, c'est d'abord le désordre du regard du spectateur, de quelqu'un qui découvre un amas de photos détachées, sans légende ni date. On se souvient du narrateur de Rue des boutiques obscures, lorsqu'un exilé russe, Stioppa de Djagoriew, lui remet entre les mains une boîte rouge remplie de photos, et les découvre avec lui. Le thème est différent, puisque

\footnotetext{
${ }^{6}$ Le texte intégral du passage se trouve reproduit en annexe, où les huit photographies décrites sont numérotées, numérotation qui est reprise dans le texte.
} 
parmi ces photos d'inconnus, le narrateur espère trouver son propre portrait, mais l'effet est le même : un sentiment de vertige, de dépaysement devant un passé qui reste flou. ${ }^{7}$ Et dans les deux cas, le narrateur a reçu ces photos des mains d'un tiers (Serge Klarsfeld dans Dora Bruder, Stioppa dans Rue des boutiques obscures) et c'est à lui d'en démêler les fils complexes.

Cependant, l'ordre de ces photos d'avant-guerre de Dora Bruder reproduit-il uniquement l'ordre contingent du regard qui les découvre pour la première fois? $\mathrm{Ce}$ va-et-vient entre petite enfance et adolescence reflète plutôt l'ordre du récit de Modiano, fait de multiples couches temporelles s'enchevêtrant sans cesse. Parfois, dans une même page du récit, on glisse de 1941 à 1996, à 1957, 1965, 1988... Ce procédé permet à Modiano de fondre sa vie avec celle de Dora Bruder, et avec celle de son propre père. Dès lors, ce qui compte, ce n'est pas tant l'histoire elle-même, mais c'est le récit de l'enquête, le compte rendu de la découverte des bribes de l'histoire de Dora. Et partant, l'ordre qui prédomine, ce n'est pas l'ordre des faits, des événements de la vie de celle-ci, mais l'ordre dans lequel ils sont remémorés, et parfois rêvés par l'auteur, à l'aide entre autre de photographies. ${ }^{8}$

Mais s'il n'y a pas d'histoire cohérente de Dora à tirer de ces photos, apportentelles du moins une réponse aux questions du narrateur? On peut se le demander. Prenons par exemple la première photo, celle du mariage des parents : «Ils sont assis, accoudés à une sorte de guéridon. Elle est enveloppée d'un grand voile blanc qui semble noué sur le côté gauche de son visage et qui traîne jusqu'à terre. » Ici, Modiano décrit, sur un ton neutre, ce qu'il voit, ni plus ni moins : la position exacte des mariés, les vêtements qu'ils portent, mais rien de plus. Pour les photos qui suivent, cette description lapidaire est complétée par quelques hypothèses quant à l'âge de Dora et au décor : «Une photo de Dora, prise certainement à l'occasion d'une distribution des prix. Elle a douze ans environ, elle porte une robe et des socquettes blanches. Elle tient dans la main droite un livre. Ses cheveux sont entourés d'une petite couronne dont on dirait que ce sont des fleurs blanches. Elle a posé sa main gauche sur le rebord d'un grand cube blanc ornementé de barres noires aux motifs géométriques, et ce cube blanc doit être là pour le décor. » (no. 3) Dans une des photos suivantes, il perce à jour les illusions du studio de photographe de l'époque : «Une autre photo de Dora et de sa mère : Dora a environ douze ans, les cheveux plus courts que sur la photo précédente. Elles sont debout devant ce qui semble un vieux mur, mais qui doit être le panneau du photographe.» (no. 5)

Pourtant, Modiano reste sensible à ce que Mieke Bal a appelé la «platitude » de la photographie : une photo n'est rien de plus que ce qu'elle donne à voir, c'est une image à deux dimensions, sans profondeur, sans épaisseur, c'est « un mirage de profondeur » (Bal 1997, p. 10). C'est pourquoi on ne saurait en tirer plus que ce qu'on voit. Par exemple dans le cas de la photo de Cécile Bruder « devant ce qui semble un pavillon de banlieue. [...] Elle est assise sur le bord de trois marches en ciment. Elle porte une robe claire d'été. Au fond, la silhouette d'un enfant, de dos, les jambes et les bras nus, en tricot noir ou en maillot de bain. Dora? Et la façade d'un autre pavillon derrière une

\footnotetext{
7 "Ça ne vous fait rien si nous arrêtons? J'ai la tête qui tourne...", dit Stioppa (Modiano 1978, p. 37)

${ }^{8}$ Comme je l'ai montré en détail ailleurs, cf. Perec, Modiano, Raczymow, 2008, p. 100.
} 
barrière de bois, avec un porche et une seule fenêtre à l'étage. Où cela peut-il être?» (no. 7) Rien, aucun grossissement de la photographie ou autre procédé technique, ne permettra de dire s'il s'agit de Dora, comme l'auteur —et le lecteur avec lui-aimerait à le croire, ou d'identifier les lieux. L'ignorance de Modiano reste entière, il ne tente pas de la combler. Mais ici encore, cette lacune vient à point nommé renforcer l'image qu'il veut donner de Dora : non pas une image nette, positive, d'un être qu'on connaît à fond, mais une image floue, une simple sihouette, ou même une apparition sortant de l'ombre, comme dans la photographie suivante, la dernière : «Une photo plus ancienne de Dora seule, à neuf ou dix ans. On dirait qu'elle est sur un toit, juste dans un rayon de soleil, avec de l'ombre tout autour.» (no. 8). Ce subtil clair-obscur convient parfaitement pour évoquer Dora qui, de plusieurs manières, est « une fugitive». Dans la vie comme dans le récit, elle surgit un instant de l'ombre pour y être, en fin de compte, replongée (c'est là aussi son « secret», que l'auteur se garde bien de violer).

Dora comme une apparition, une ombre : c'est ainsi qu'elle apparaît sur ces deux photographies. Et c'est précisément de cette manière aussi qu'elle est décrite dans le récit. Lorsque Modiano part sur ses traces, lorsqu'il s'attarde dans les lieux qu'elle a fréquentés, il ressent, de manière insistante, la présence de son absence. Il est hanté par son fantôme, son spectre, dans ces lieux qui «gardent une légère empreinte des personnes qui les ont habités. Empreinte : marque en creux ou en relief. Pour Ernest et Cécile Bruder, pour Dora, je dirai : en creux.» (Dora Bruder pp. 28-29). Ce passage précède les photographies écrites de quelques pages seulement. Celles-ci viennent donc non pas combler le vide, mais l'accentuer d'une autre manière.

La photographie en prose, dans Dora Bruder, va donc dans le sens de la conception barthésienne de la photographie, si souvent citée. Chez Modiano, la photo est certes un index : qu'elle soit réelle ou inventée (comme dans les autres romans de Modiano), elle fonctionne comme une preuve tangible que «ça a été » (Barthes 1980, p. 120), que Dora et ses parents ont bel et bien existé, elle est une «pièce à conviction», selon le terme de Modiano. Mais comme index, elle signifie aussi que son référent, que Dora n'est plus, qu'elle a disparu, dans tous les sens du terme. Ces photos expriment donc bien le mystère, le double message que Barthes discerne dans la photo : la photographie est entre la vie et la mort, à plus forte raison dans le cas de Dora puisqu'elle a péri prématurément, en déportation. C'est ce savoir qui rend ces photos si poignantes, pour Modiano comme pour le lecteur.

Dans le cas de Dora-mais cela vaut tout autant pour les autres photographies en prose qui apparaissent dans les romans de Modiano-c'est ce dernier versant de la photo qui domine : celui de la disparition. La photographie, dans son oeuvre, confirme le fragile statut ontologique des personnes : elles sont toujours déjà en voie de disparition. On se demande même si elles ont jamais eu une existence ferme, si elles sont faites de chairs et d'os. Ce sont de minces silhouettes, à l'identité floue, toujours sur le point de se volatiliser. Ainsi cette photo de l'enfant vue de dos (no. 7) renvoie à tant d'autres photos en prose dans son œuvre. Dans Photofictions, Roger-Yves Roche analyse Chien de printemps, roman où la photographie joue un rôle prépondérant, puisque le narrateur y enquête sur un photographe. Or devant les œuvres du photographe Jansen, Roche aboutit ici à une conclusion semblable : «Des photographies qui ne transmettent rien, des photographies dans lesquelles fantômes et spectres n'en finissent pas d'errer, une littérature devenue 'susceptible'. Et 
pourtant, des photographies que l'on ne cesse de transmettre, de s'échanger, des photographies autour desquelles on parle, à l'ombre desquelles on échafaude des hypothèses, on construit des semblants de récit. » (Roche 2009, p. 112). Paradoxalement, en effet, malgré le manque inhérent à la photographie, ou peut-être en raison de celui-ci, il faut s'acharner à les décrire en détail, car la description méticuleuse, noir sur blanc, est la seule manière de fixer des photographies somme toute périssables, et de leur conférer un sens.

La photo, chez Modiano, loin de faire resurgir un être, le montre donc plongé dans l'absence, dans le flou. Voilà qui explique peut-être que, dans ce premier passage du moins, il ne tente d'aucune façon de décrire les traits des personnes portraiturées sur ces photos. Il se limite à mentionner leur position et leurs vêtements, le décor, il fait des suppositions sur les circonstances, mais il passe sur leur expression, sur tout ce qui pourrait faire référence à une intériorité. On pourrait expliquer cette extrême retenue par le respect du « secret » de Dora : à la fin du récit, il constate que la fugue de Dora restera à jamais mystérieuse et appelle à respecter ce secret et par extension, le secret de sa vie, de son être. Comme si tout ce qui touchait à son intériorité était d'une certaine manière sacré, intouchable. Il ne faut pas oublier cependant que ce flou, cette absence de traits particuliers appartient en propre à la photographie, telle que la conçoit Modiano.

Dans la description de ces huit photos, il n'invente rien, il se retient même de décrire autre chose que ces photos dans leur 'platitude'. Rien qui puisse révéler l'état d'esprit de Dora ou de ses parents. Pourtant, il y a un élément qui nous en parle indirectement, c'est le blanc. Car à y regarder de près, tout est blanc, dans ces deux pages : la mariée d'abord, cela va de soi, avec son «grand voile blanc », et le marié avec son nœud à papillon blanc. Ensuite Dora à douze ans, en robe et socquettes blanches, couronnée de blanc, la main posée sur un cube blanc (no. 3). L'auteur suppose qu'il s'agit d'une distribution des prix. En mettant en valeur le blanc, dans toutes ces photos, il fait référence indirectement à l'innocence qui entoure le mariage et la petite enfance. Mais il y a plus : toutes ces photos appartiennent à une période de bonheur innocent, naïf presque, à laquelle les persécutions et les déportations mettront bientôt fin. C'est cela qu'il lui importe de souligner en mettant en valeur les touches de blanc, dans ces photos : Dora à 12 ans avec une robe noire à col blanc (no. 5), Dora et sa mère en chemisier blanc (no. 6)... Et enfin les deux dernières photos, les seules à ne pas être prises en studio. D'abord Cécile «en robe claire d'été » avec au fond la silhouette d'un enfant en maillot de bain : du blanc on glisse vers le clair, vers l'été qui renforce l'atmosphère de bonheur innocent (no. 7). Ensuite Dora sur un toit, juste dans un rayon de soleil : «Elle porte une blouse et des socquettes blanches, elle tient son bras gauche replié sur sa hanche et elle a posé le pied droit sur le rebord de béton de ce qui pourrait être une grande cage ou une grande volière, mais on ne distingue pas, à cause de l'ombre, les animaux ou les oiseaux qui y sont enfermés. Ces ombres et ces taches de soleil sont celles d'un jour d'été. » (no. 8) Ainsi, grâce à la mise en texte, toute cette série de photos ne forme qu'une seule métaphore filée du blanc, symbole transparent de jeunesse, d'innocence et d'insouciant bonheur. 


\section{Un regard d'outre-tombe}

Par son atmosphère d'innocence, ce premier ensemble est en contraste absolu avec le second, qui consiste en une seule photographie en prose, celle de Dora adolescente avec sa mère et sa grand-mère maternelle. Comme l'observe Modiano, c'est «une photo qui tranche sur celles que j'avais déjà rassemblées.» Finie l'enfance, fini le blanc, maintenant c'est la gravité, la tristesse même qui dominent. La robe blanche est brutalement devenue une robe noire. Afin de comprendre ce contraste, il faut ici encore prendre en compte le contexte où Modiano place cette photographie écrite. Une soixantaine de pages après le premier ensemble de photos en prose, nous sommes ici au cœur du drame : c'est l'hiver 1941-1942, le père de Dora a déjà été arrêté, Dora fait deux fugues successives, l'étau se resserre:

J'ai pu obtenir il y a quelques mois une photo de Dora Bruder, qui tranche sur celles que j'avais déjà rassemblées. Sans doute la dernière qui a été prise d'elle. Son visage et son allure n'ont plus rien de l'enfance qui se reflétait dans toutes les photos précédentes à travers le regard, la rondeur des joues, la robe blanche d'un jour de distribution des prix... Je ne sais pas à quelle date a été prise cette photo. Certainement en 1941, l'année où Dora était pensionnaire au Saint-Cœur-de-Marie, ou bien au début du printemps 1942, quand elle est revenue, après sa fugue de décembre, boulevard Ornano.

Elle est en compagnie de sa mère et de sa grand-mère maternelle. Les trois femmes sont côte à côte, la grand-mère entre Cécile Bruder et Dora. Cécile Bruder porte une robe noire et les cheveux courts, la grand-mère une robe à fleurs. Les deus femmes ne sourient pas. Dora est vêtue d'une robe noire-ou bleu marine - et d'une blouse à col blanc, mais cela pourrait être aussi un gilet et une jupe-la photo n'est pas assez nette pour s'en rendre compte. Elle porte des bas et des chaussures à brides. Ses cheveux mi-longs lui tombent presque jusqu'aux épaules et sont ramenés en arrière par un serre-tête, son bras gauche est le long du corps, avec les doigts de la main gauche repliés et le bras droit caché par sa grand-mère. Elle tient la tête haute, ses yeux sont graves, mais il flotte sur ses lèvres l'amorce d'un sourire. Et cela donne à son visage une expression de douceur triste et de défi. Les trois femmes sont debout devant le mur. Le sol est dallé, comme le couloir d'un lieu public. Qui a bien pu prendre cette photo? Ernest Bruder? Et s'il ne figure pas sur cette photo, cela veut-il dire qu'il a déjà été arrêté? En tout cas, il semble que les trois femmes aient revêtu leurs habits du dimanche, face à cet objectif anonyme.

Dora porte-t-elle la jupe bleu marine indiquée sur l'avis de recherche? (Dora Bruder, pp. 90-91)

A première vue, cette image en prose est une description extrêmement factuelle, détaillée, de la photo en question (Fig. 5). Surtout le deuxième paragraphe s'ingénie à décrire le plus précisément possible la position des personnes portraiturées et leurs vêtements. Mais Modiano fait immédiatement une série de suppositions. La datation tout d'abord : avec un certain aplomb— «sans doute», c'est-à-dire "probablement»-il pose que cette photo est «la dernière qui a été prise d'elle. » Rien de 
Fig. 5 Dora avec sa mère et sa grand-mère (Dora Bruder pp. 90-91). Photo reproduite avec l'autorisation de P. Modiano

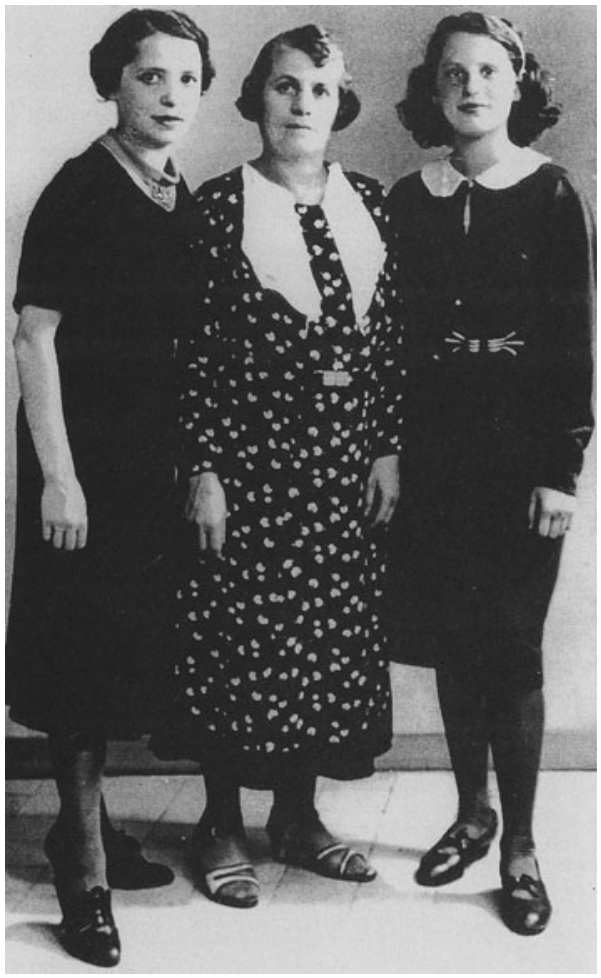

moins sûr cependant : qui sait quelles photos Serge Klarsfeld pourra encore lui procurer? Mais cette hypothèse convient bien à l'atmosphère générale de fatalité dont il revêt cette photo. Si cette photo est la dernière, elle doit avoir été prise pendant les mois qui précèdent la déportation. Là encore, tout en avouant son ignorance, il semble sûr de son affaire : «Je ne sais pas à quelle date cette photo a été prise. Certainement en 1941, l'année où Dora était pensionnaire au Saint-Cœurde-Marie, ou bien au début du printemps 1942, quand elle est revenue, après sa fugue de décembre, boulevard Ornano. » (je souligne). Ce qui plaide en faveur de cette datation globale, c'est que la photo montre Dora adolescente, jeune femme même. Par trois fois, le texte le souligne en disant «les trois femmes». Dora n'est plus une enfant, elle a pris sa place de femme face à sa mère et à sa grand-mère.

En outre, pour Modiano, les vêtements de Dora viennent confirmer ce contraste avec les photos précédentes elle porte maintenant une robe sombre (comme sa mère et sa grand-mère) et une blouse à col blanc. Comme si les trois femmes étaient prématurément en deuil d'elles-mêmes! Et le flou de cette photo lui permet de faire une supposition encore plus intéressante, de son point de vue : est-ce que, au lieu d'une robe noire, ce ne serait pas une jupe bleu marine, la jupe bleu marine de l'annonce de France Soir? Voilà qui conviendrait parfaitement à sa conviction que ce serait ici la dernière photo de Dora, prise juste avant sa deuxième fugue et donc son arrestation... On voit que les suppositions s'accumulent, allant toutes dans le 
sens de la fatalité. Selon Modiano, nous aurions ici le portrait d'une Dora déjà fugueuse, le portrait correspondant à l'annonce qui a déclenché l'enquête.

Tous ces éléments se rejoignent dans l'expression des trois femmes, qui cette fois-ci est bel et bien mentionnée : la mère et la grand-mère ne sourient pas. Quant à Dora, dans son visage, Modiano lit deux éléments contrastants : d'une part la tristesse, la gravité, de l'autre la fierté, le défi même. Cette gravité et cette «douceur triste », on les rattacherait en première instance aux tribulations que les femmes ont déjà vécues : persécutions, peut-être arrestation du père. Mais pour l'auteur, comme pour le lecteur, cette tristesse renvoie aussi à l'avenir, à tout ce qui les attend encore, c'est-à-dire la déportation et la mort. Cette supposition, que Modiano laisse entièrement implicite, explique le caractère poignant de cette photographie. Il la renforce en insistant sur le décor : les trois femmes seraient debout devant un mur, sur un sol dallé, «comme dans un lieu public». Le sol dallé de blanc est nettement visible sur la photographie, le mur se réduit à une marche. Mais l'observation de Modiano suggère, là encore implicitement, que dès cette photo, elles sont comme arrachées à leur environnement familier-ce qui anticipe sur leur déportation. Elles sont littéralement au pied du mur!

Essayons de cerner d'un peu plus près ce regard du spectateur. Cette photo, comme les autres d'ailleurs, appartient à ce qu'on appellera, avec Marianne Hirsch, les 'Holocaust photographs' (Hirsch 2001). Il ne s'agit pas uniquement des photos des déportations et des camps, mais aussi de toute la réserve de photos de famille des victimes. Ce n'est pas leur thème qui lie ces photos à la Shoah, mais leur contexte (Hirsch 1997). Elles portent en elles un contraste entre leur thème et leur contexte : alors qu'elles sont pleines de vie, d'une vie 'normale', ces photos d'anniversaires ou d'autres réunions de famille baignent dans une atmosphère de mort pour le spectateur qui, lui, connaît le sort de ces personnes. Ces photos, comme Susan Sontag l'avait montré, sont porteuses d'une «ironie posthume», sensible uniquement à ceux qui vivent après, et qui possèdent un savoir a posteriori (Sontag 1973, p. 63). Or, inconsciemment, ceux-ci projettent ce savoir sur ces photos, transformant les personnes portraiturées elles aussi en témoins qui savent, qui supputeraient déjà le sort fatal qui les attend, d'où leur air mélancolique. Alors que ces personnes ignorent tout de leur sort. Un exemple bien connu de ce mécanisme, et mentionné par Sontag, est le célèbre album de Roman Vishniac, A vanished world, publié en 1947 (Vishniac 1983). Comme on sait, entre 1936 et 1939, donc juste avant la Shoah, ce photographe avait parcouru toute l'Europe de l'Est pour photographier la vie dans les shtetls et ghettos, conscient que celle-ci était condamnée à périr. Même le titre-《un univers disparu»—est un bel exemple d'une telle ironie posthume, il incite le lecteur à considérer cet univers-alors bien vivant-comme un univers disparu (ce qui est correct en 1947). ${ }^{9}$

Dans la photographie en prose des trois femmes, il y a certes un tel regard d'outre-tombe, c'est celui du spectateur, de Modiano en l'occurrence. La dominance du noir et le regard grave des femmes le suggèrent. De plus, il s'agit ici d'un portrait de trois générations de femmes. Il exprime que, pendant deux générations au moins,

\footnotetext{
${ }^{9}$ Nous avons rencontré plus haut $(\$ 1)$ un autre exemple de ce mécanisme, c'est l'interprétation par Denise Cima de la photographie de couverture de l'édition Folio de Dora Bruder.
} 
la procréation a suivi son cours, mais avec Dora, qui sera déportée et assassinée, voilà que le cycle des générations s'arrête... La lignée est interrompue. Celle de la famille Bruder et par extension, celle du peuple juif tout entier. Par sa mise en scène de ces trois générations, cette photographie écrite s'insère donc parfaitement dans la commémoration des autres Juifs parisiens déportés, dans la suite de cette section et les suivantes. Mais là encore, seul un regard a posteriori, posthume même, celui de l'auteur, peut faire un tel rapport. Pourtant, l'auteur insère aussi une tout autre note : Dora a beau être grave et triste, cela ne l'empêche pas de tenir «la tête haute», et son visage a une expression «de défi». C'est que Dora, aux yeux de Modiano, est loin d'être une victime passive. Elle est aussi une adolescente rebelle, une fugueuse qui à sa manière, même si elle ne réussit pas à éviter l'arrestation, garde son indépendance d'esprit. Cela nous ramène au registre autobiographique, lui aussi riche en résonances, dans le récit.

Pourquoi, en conclusion, Modiano a-t-il opté pour l'ekphrasis, dans Dora Bruder, et non pour la reproduction des photographies décrites? Une raison en est certainement la retenue qu'il observe, conscient de n'avoir reconstruit que des fragments épars de la vie de Dora. Il s'agit, comme il le dit lui-même, de respecter le «secret» de Dora, de sa vie. Mais la raison principale est d'ordre littéraire, c'est la fidélité au récit tel que le pratique Modiano. Comme dans ses autres textes, l'enquête est présentée par un narrateur qui en est le focalisateur unique : tout, y inclus les photos, est vu par son regard, et par là même passe par le filtre de sa sensibilité. C'est un regard paradoxal : d'une part il observe une extrême retenue, il n'invente rien à propos des photos, il les décrit de manière très factuelle, sans essayer d'en tirer une histoire cohérente. Mais de l'autre, cette retenue fait justement que Dora reste un personnage flou, une ombre et par là même, elle s'insère parfaitement dans l'univers romanesque de Modiano, jusqu'à en devenir un personnage typique. Ce flou permet à Modiano de dépeindre subtilement les variations d'atmosphère de ces photos : innocence, bonheur d'abord, appréhension de l'avenir ensuite. Or toutes ces touches ne peuvent être apportées que par la photographie en prose, par l'écriture donc, la photographie brute ne saurait les apporter.

Open Access This article is distributed under the terms of the Creative Commons Attribution Noncommercial License which permits any noncommercial use, distribution, and reproduction in any medium, provided the original author(s) and source are credited.

\section{Annexe: Dora Bruder, pp. 31-33}

«Quelques photos de cette époque. (1) La plus ancienne, le jour de leur mariage. Ils sont assis, accoudés à une sorte de guéridon. Elle est enveloppée d'un grand voile blanc qui semble noué sur le côté gauche de son visage et qui traine jusqu'à terre. Il est en habit et porte un nœud papillon blanc. (2) Une photo avec leur fille Dora. Ils sont assis, Dora debout entre eux : elle n'a pas plus de deux ans. (3) Une photo de Dora, prise certainement à l'occasion d'une distribution des prix. Elle a douze ans environ, elle porte une robe et des socquettes blanches. Elle tient dans la main droite 
un livre. Ses cheveux sont entourés d'une petite couronne dont on dirait que ce sont des fleurs blanches. Elle a posé sa main gauche sur le rebord d'un grand cube blanc ornementé de barres noires aux motifs géométriques, et ce cube blanc doit être là pour le décor. (4) Une autre photo, prise dans le même lieu, à la même époque et peut-être le même jour : on reconnaît le carrelage du sol et ce grand cube blanc aux motifs géométriques sur lequel est assise Cécile Bruder. Dora est debout à sa gauche dans une robe à col, le bras gauche replié devant elle afin de poser la main sur l'épaule de sa mère. (5) Une autre photo de Dora et de sa mère : Dora a environ douze ans, les cheveux plus courts que sur la photo précédente. Elles sont debout devant ce qui semble un vieux mur, mais qui doit être le panneau du photographe. Elles portent toutes les deux une robe noire et un col blanc. Dora se tient légèrement devant sa mère et à sa droite. (6) Une photo de forme ovale où Dora est un peu plus âgée-treize, quatorze ans, les cheveux plus longs-et où ils sont tous les trois comme en file indienne, mais le visage face à l'objectif : d'abord Dora et sa mère, toutes deux en chemisier blanc, et Ernest Bruder, en veste et cravate. (7) Une photo de Cécile Bruder, devant ce qui semble un pavillon de banlieue. Au premier plan, à gauche, une masse de lierre recouvre le mur. Elle est assise sur le bord de trois marches en ciment. Elle porte une robe claire d'été. Au fond, la silhouette d'un enfant, de dos, les jambes et les bras nus, en tricot noir et en maillot de bain. Dora? Et la façade d'un autre pavillon derrière une barrière de bois, avec un porche et une seule fenêtre à l'étage. Où cela peut-il être?

(8) Une photo plus ancienne de Dora seule, à neuf ou dix ans. On dirait qu'elle est sur un toit, juste dans un rayon de soleil, avec de l'ombre tout autour. Elle porte une blouse et des socquettes blanches, elle tient son bras gauche replié sur sa hanche et elle a posé le pied droit sur le rebord de béton de ce qui pourrait être une grande cage ou une grande volière, mais on ne distingue pas, à cause de l'ombre, les animaux ou les oiseaux qui y sont enfermés. Ces ombres et ces taches de soleil sont celles d'un jour d'été. »

\section{Bibliographie}

Bal, M. (1997). Images littéraires ou comment lire visuellement Proust. Montréal: XYZ. Barthes, R. (1980). La chambre claire. Paris: Gallimard Seuil.

Cima, D. (2003). Etude sur Dora Bruder de Patrick Modiano. Paris: Ellipses.

De Gaudemar, A. (1997). La dernière fugue de Dora. Libération, 3 avril.

Frain, I. (1997). Patrick Modiano, l'éternel préoccupé de 1'Occupation. Paris-Match, 17 avril.

Hirsch, M. (1996). Past lives: Postmemories in Exile. Poetics Today, 17(4), 659-686.

Hirsch, M. (1997). Family frames. Photography, narrative and postmemory. Cambridge, MA: Harvard University Press.

Hirsch, M. (2001). Surviving images: Holocaust photographs and the work of postmemory. The Yale Journal of Criticism, 14(1), 5-37.

Klarsfeld, S. (1978). Mémorial de la déportation des Juifs de France. Paris: Association pour le jugement des criminels nazis qui ont opéré en France.

Klarsfeld, S. (2001). La Shoah en France, vol. 4: Le Mémorial des enfants juifs déportés. Paris: Fayard.

Modiano, P. (1978). Rue des boutiques obscures. Paris: Gallimard Nrf.

Modiano, P. (1994). Avec Klarsfeld contre l'oubli. Libération, 2 novembre, 8.

Modiano, P. (1997). Dora Bruder. Paris: Gallimard Nrf. 
Modiano, P. (1999a). Dora Bruder. Paris: Gallimard Folio.

Modiano, P. (1999b). Dora Bruder (J. Kilmartin, Trans.). Berkeley, Los Angeles, Londres: University of California Press.

Modiano, P. (2001). Livret de famille. Paris: Gallimard Folio.

Morris, A. (2006). Avec Serge Klarsfeld contre l'oubli. Patrick Modiano's Dora Bruder. Journal of European Studies, 36(3), 269-293.

Roche, J.-Y. (2009). Photofictions. Perec, Modiano, Duras, Goldschmidt, Barthes. Villeneuve d'Ascq: Presses universitaires du Septentrion.

Schulte Nordholt, A. (2008). Perec, Modiano, Raczymow. La génération d'après et la mémoire de la Shoah. Rodopi: Amsterdam.

Sontag, S. (1973). On photography. New York: Farrar, Straus and Giroux.

Vishniac, R. (1983). A vanished world. Amsterdam: Meulenhoff/Landshoff. [New York: Shocken Books, 1947]. 